\title{
Exact Solution of Linear and Nonlinear Wave Equations by Double Laplace and Iterative Method
}

\author{
Adesina K. Adio ${ }^{1}$, Wumi S. Ajayi ${ }^{2}$, Olabode O. Bamisile ${ }^{1}$ \& Babatunde T. Akanbi ${ }^{1}$ \\ ${ }^{1}$ Department of Basic Sciences, School of Science and Technology, Babcock University, Nigeria \\ ${ }^{2}$ Department of Computer Sciences, School of Computing and Engineering, Babcock University, Nigeria \\ Correspondence: Adesina K. Adio, Department of Basic Sciences, School of Science and Technology, Babcock \\ University, Nigeria. E-mail: adioa@babcock.edu.ng
}

Received: July 4, 2019 Accepted: August 20, 2019 Online Published: September 16, 2019

doi:10.5539/jmr.v11n5p33 URL: https://doi.org/10.5539/jmr.v11n5p33

\begin{abstract}
A coupling of double Laplace transform with Iterative method is used to solve linear and nonlinear wave equations subject to initial and boundary conditions. The iteration process leads to disappearance of noise terms and exact solution is obtained at first iteration. Through several examples, the convenience and efficiency of the method is demonstrated, showing its usefulness to overcome difficulties associated with some existing techniques.
\end{abstract}

Keywords: double Laplace transform, Inverse double Laplace transform, iterative method, wave equations

\section{Introduction}

The wave equation is known as one of three fundamental equations in mathematical physics and occurs in many branches of physics, applied mathematics, and engineering (Eltayeb \& Kiliciman, 2013).

Many attempts have been made to develop analytic and approximate methods to solve the linear and nonlinear wave equations (Wazwaz, 2007, 2001, Sadighi \& Ghanji, 2007, Al-Jawary, 2015). Although such methods have been successfully applied but some difficulties have appeared, for examples, in calculating Adomian polynomials to handle the nonlinear terms in ADM (Wazwaz, 2001), calculation of Lagrange multiplier in VIM (Wazwaz, 2007, Sadighi \& Ghanji, 2007), also application of homotopy perturbation method requires construction of a homotopy and solving the corresponding algebraic equations.

In this paper, double Laplace transform combined with iterative method developed by Dhunde and Waghmare is used to solve the linear and nonlinear wave equation. The method which demonstrates simplicity in its principles have been used to solve non-linear telegraph equation (Dhunde \& Waghmare, 2016a) and non-linear Klein-Gordon equation (Dhunde \& Waghmare, 2016b).

The method is simple to understand and easy to implement. It has no specific requirements such as linearization, perturbation, small parameters etc. for nonlinear operators as required by some existing techniques.

This paper has been organized as follows. Section 2 is about the theoretical background of the work. In section 3 , the double Laplace transform coupled with iterative method is applied to a generalized non-linear wave equation, while Section 4 is about application to linear and nonlinear wave equations and finally in section 5 the discussion of result is presented.

\section{Theoretical Background}

Linear and nonlinear wave equations are given by (Wazwaz, 2001, Al-Jawary, 2015)

$$
u_{t t}(x, t)=u_{x x}(x, t)+f(u) \quad ; \quad 0<x<L
$$

and

$$
u_{t t}(x, t)=u_{x x}(x, t)+F(u)+g(x, t) ; 0<x<L
$$

respectively.

The functions $f(u), F(u)$ and $g(x, t)$ are linear, nonlinear and source functions respectively.

The wave equation plays an important role in various physical problems. Study of the wave equation is needed in diverse 
areas of engineering and scientific applications.

Recall the following definitions (Eltayeb \& Kiliciman, 2013, Eltayeb, 2017).

The double Laplace transform is defined by

$$
L_{x} L_{t}[f(x, s)]=F(p, s)=\int_{0}^{\infty} e^{-p x}\left(\int_{0}^{\infty} e^{-s t} f(x, t) d t\right) d x,
$$

Whenever the integral exist, where $f(x, t)$ is a function of two variables $x$ and $t$ defined in the positive quadrant of the $x t$-plane, $x, t>0$ and $p, s$ are complex numbers.

Double Laplace transform for second partial derivative with respect to $x$ is given by

$$
L_{x x}\left[\frac{\partial^{2} f(x, t)}{\partial x^{2}}\right]=p^{2} F(p, s)-p F(0, s)-\frac{\partial F}{\partial x}(0, s),
$$

And double Laplace for second partial derivative with respect to $t$ is given by

$$
L_{t t}\left[\frac{\partial^{2} f(x, t)}{\partial t^{2}}\right]=s^{2} F(p, s)-s F(p, 0)-\frac{\partial F}{\partial t}(p, 0),
$$

from (2.3); we deduce (Dhunde \& Waghmare, 2016)

$$
L_{x} L_{t}[f(x) g(t)]=\bar{f}(p) \bar{g}(s)=L_{x}[f(x)] L_{t}[g(t)]
$$

Further the double Laplace transform of second order partial derivatives are given by

$$
\begin{aligned}
& L_{x} L_{t}\left[\frac{\partial^{2} f(x, t)}{\partial x^{2}}\right]=p^{2} \bar{f}(p, s)-p \bar{f}(0, s)-\bar{f}_{x}(0, s) \\
& L_{x} L_{t}\left[\frac{\partial^{2} f(x, t)}{\partial t^{2}}\right]=s^{2} \bar{f}(p, s)-s \bar{f}(p, 0)-\bar{f}_{t}(p, 0)
\end{aligned}
$$

The inverse double Laplace transform

$L_{x}{ }^{-1} L_{t}{ }^{-1}[\bar{f}(p, s)]=f(x, t)$ is defined by the complex double integral formula

$$
L_{x}{ }^{-1} L_{t}{ }^{-1}[\bar{f}(p, s)]=f(x, t)=\frac{1}{2 \pi i} \int_{c-i \infty}^{c+i \infty} e^{p x} d p\left(\frac{1}{2 \pi i} \int_{d-i \infty}^{d+i \infty} e^{s t} \bar{f}(p, s) d s\right)
$$

Where $\bar{f}(p, s)$ must be an analytical function for all $p$ and $s$ in the region defined by the inequalities $\operatorname{Re} p \geq c$ and Re $s \geq d$, where $c$ and $d$ are real constants to be chosen suitably.

\section{Double Laplace Transform and Iterative Method}

Now consider the non linear wave equation

$$
\frac{\partial^{2} u(x, t)}{\partial t^{2}}=\frac{\partial^{2} u(x, t)}{\partial x^{2}}+F u(x, t)+g(x, t) ; \quad 0<x<L
$$

with the initial conditions

$$
u(x, 0)=h_{1}(x) ; \quad \frac{\partial u}{\partial t}(x, 0)=h_{2}(x)
$$

and the boundary conditions

$$
u(0, t)=h_{1}(t) ; \quad u_{x}(0, t)=h_{2}(t)
$$

where $u(x, t)$ represents the wave displacement at position $x$ and time $t, F(u)$ is the nonlinear force where $\frac{\partial F}{\partial u} \geq 0 ; g(x, t) ; h_{1}(x) ; h_{2}(x) ; h_{1}(t)$ and $h_{2}(t)$ are known functions.

Application of double Laplace transform on (3.1) gives 


$$
\begin{gathered}
s^{2} \bar{u}(p, s)-s \bar{u}(p, 0)-\bar{u}_{t}(p, 0)=p^{2} \bar{u}(p, s)-p \bar{u}(0, s)-\bar{u}_{x}(0, s)+ \\
L_{x} L_{t}[F(u(x, t))+g(x, t)]
\end{gathered}
$$

Application of single Laplace transform to (3.2) and (3.3) leads to

$$
\bar{u}(p, 0)=\bar{h}_{1}(p) ; \bar{u}_{t}(p, 0)=\bar{h}_{2}(p) ; \bar{u}(0, s)=\bar{h}_{1}(s) \text { and } \bar{u}_{x}(0, s)=\bar{h}_{2}(s)
$$

Substituting (3.5) in (3.4) and simplifying; we have

$$
\bar{u}(p, s)=\frac{s \bar{h}_{1}(p)+\bar{h}_{2}(p)-p \bar{h}_{1}(s)-\bar{h}_{2}(s)}{s^{2}-p^{2}}+\frac{1}{s^{2}-p^{2}}\left\{L_{x} L_{t}[F(u(x, t))+g(x, t)]\right\}
$$

Application of inverse double Laplace transform to (3.6) leads to

$$
\begin{aligned}
u(x, t)= & L^{-1}{ }_{x} L^{-1} t\left[\frac{s \bar{h}_{1}(p)+\bar{h}_{2}(p)-p \bar{h}_{1}(s)-\bar{h}_{2}(s)}{s^{2}-p^{2}}\right] \\
& +L^{-1}{ }_{x} L^{-1}{ }_{t}\left[\frac{1}{s^{2}-p^{2}}\left\{L_{x} L_{t}[F(u(x, t))+g(x, t)]\right\}\right]
\end{aligned}
$$

Applying the Iterative method

Substituting (3.8) in (3.7), we obtain

$$
u(x, t)=\sum_{i=0}^{\infty} u_{i}(x, t)
$$

$$
\begin{aligned}
\sum_{i=0}^{\infty} u_{i}(x, t) & =L^{-1}{ }_{x}{ }^{-1}{ }_{t}\left[\frac{s \bar{h}_{1}(p)+\bar{h}_{2}(p)-p \bar{h}_{1}(s)-\bar{h}_{2}(s)}{s^{2}-p^{2}}\right] \\
& +L^{-1}{ }_{x} L^{-1}{ }_{t}\left[\frac{1}{s^{2}-p^{2}}\left\{L_{x} L_{t}\left[F\left(\sum_{i=0}^{\infty} u_{i}(x, t)\right)+g(x, t)\right]\right\}\right]
\end{aligned}
$$

The nonlinear term $F$ is decomposed as

Put (3.10) in (3.9) to obtain

$$
F\left(\sum_{i=0}^{\infty} u_{i}(x, t)\right)=F\left(u_{0}(x, t)\right)+\sum_{i=1}^{\infty}\left[F\left(\sum_{k=0}^{i} u_{k}(x, t)\right)-F\left(\sum_{k=0}^{i-1} u_{k}(x, t)\right)\right]
$$

$$
\begin{gathered}
\sum_{i=0}^{\infty} u_{i}(x, t)=L^{-1}{ }_{x} L^{-1}{ }_{t}\left[\frac{s \bar{h}_{1}(p)+\bar{h}_{2}(p)-p \bar{h}_{1}(s)-\bar{h}_{2}(s)}{s^{2}-p^{2}}\right] \\
+L^{-1}{ }_{x} L^{-1}{ }_{t}\left[\frac{1}{s^{2}-p^{2}}\left\{L_{x} L_{t}\left[F\left(u_{0}(x, t)\right)+g(x, t)\right]\right\}\right] \\
+L^{-1}{ }_{x} L^{-1}{ }_{t}\left[\frac{1}{s^{2}-p^{2}}\left\{L_{x} L_{t}\left[\sum_{i=1}^{\infty} F\left(\sum_{k=0}^{i} u_{k}(x, t)-F\left(\sum_{k=0}^{i-1} u_{k}(x, t)\right)\right)\right]\right\}\right]
\end{gathered}
$$

we then define the recurrence relations as

$$
\begin{gathered}
u_{0}(x, t)=L_{x}^{-1} L_{t}^{-1}\left[\frac{s \bar{h}_{1}(p)+\bar{h}_{2}(p)-p \bar{h}_{1}(s)-\bar{h}_{2}(s)}{s^{2}-p^{2}}\right] \\
u_{1}(x, t)=L^{-1}{ }_{x} L^{-1}{ }_{t}\left[\frac{1}{s^{2}-p^{2}}\left\{L_{x} L_{t}\left[F\left(u_{0}(x, t)\right)+g(x, t)\right]\right\}\right]
\end{gathered}
$$

and

$$
u_{n+1}(x, t)=L^{-1}{ }_{x} L^{-1}{ }_{t}\left[\frac{1}{s^{2}-p^{2}}\left\{L_{x} L_{t}\left[\sum_{i=1}^{\infty} F\left(\sum_{k=0}^{i} u_{k}(x, t)-F\left(\sum_{k=0}^{i-1} u_{k}(x, t)\right)\right)\right]\right\},\right]
$$

The recurrence relation generates the solution of (3.1) in series form given by 


\section{Applications}

$$
u(x, t)=u_{0}(x, t)+u_{1}(x, t)+u_{2}(x, t)+\ldots+u_{n}(x, t)+\ldots
$$

Here, we applied double Laplace transform couple with the iterative method to solve five examples of wave equations. The aim is to demonstrate the simplicity and efficiency of the method.

Example 4.1. Consider the inhomogenous non-linear wave equation:

$$
u_{t t}(x, t)=u_{x x}(x, t)+u+u^{2}-x t-x^{2} t^{2} ; 0<x<\pi, t>0
$$

with initial conditions

$$
u(x, 0)=0 ; \quad u_{t}(x, 0)=x,
$$

and boundary conditions

$$
u(0, t)=0 ; \quad u(\pi, t)=\pi t,
$$

applying the double Laplace transform on both sides of (4.1), we have

$$
\begin{gathered}
s^{2} \bar{u}(p, s)-s \bar{u}(p, 0)-\bar{u}_{t}(p, 0)-p^{2} \bar{u}(p, s)+p \bar{u}(0, s)+\bar{u}_{x}(0, s)= \\
L_{x} L_{t}\left[u+u^{2}-x t-x^{2} t^{2}\right]
\end{gathered}
$$

further application of single Laplace transform to conditions (4.2) and (4.3) gives

$$
\bar{u}(p, 0)=0 ; \bar{u}_{t}(p, 0)=\frac{1}{p^{2}} ; \bar{u}(0, s)=0 \text { and } \bar{u}_{x}(0, s)=\frac{1}{s^{2}}
$$

Substitute (4.5) in (4.4) and simplify to obtain

$$
\bar{u}(p, s)=\frac{1}{p^{2} s^{2}}+\frac{1}{(s-p)(s+p)} L_{x} L_{t}\left[u+u^{2}-x t-x^{2} t^{2}\right]
$$

applying inverse double Laplace transform on (4.6), we obtain

$$
u(x, t)=x t+L_{x}{ }^{-1} L_{t}{ }^{-1}\left\{\frac{1}{(s-p)(s+p)} L_{x} L_{t}\left[u+u^{2}-x t-x^{2} t^{2}\right]\right\}
$$

To apply the Iterative method, we substitute (3.8) in (4.7) and apply (3.12) - (3.14). thus the components of the solution are:

$$
\begin{gathered}
u_{0}(x, t)=x t \\
u_{1}(x, t)=L_{x}^{-1} L_{t}^{-1}\left\{\frac{1}{s^{2}-p^{2}} L_{x} L_{t}\left[u_{0}+u_{0}^{2}-x t-x^{2} t^{2}\right]\right\}=0 \\
u_{n+1}(x, t)=-L_{x}^{-1} L_{t}^{-1}\left\{\frac{1}{s^{2}-p^{2}} L_{x} L_{t}\left[\sum_{k=0}^{n}\left(u_{k}(x, t)\right)^{2}-\left(\sum_{k=0}^{n-1} u_{k}(x, t)\right)^{2}\right]\right\} ; n \geq 1 \\
u_{2}(x, t)=-L_{x}{ }^{-1} L_{t}{ }^{-1}\left\{\frac{1}{s^{2}-p^{2}} L_{x} L_{t}\left[\left(u_{0}(x, t)+u_{1}(x, t)\right)^{2}-\left(u_{0}(x, t)\right)^{2}\right]\right\} \quad=0
\end{gathered}
$$

Similarly, $u_{3}(x, t)=u_{4}(x, t)=u_{5}(x, t)=0$ and so on.

Thus we obtain the solution of (4.1) as

which is the required solution.

$$
u(x, t)=x t
$$

Example 4.2. Consider the homogenous wave equation:

$$
u_{t t}(x, t)=u_{x x}(x, t) ; 0<x<\pi, t>0
$$

with initial conditions

$$
u(x, 0)=\sin x ; \quad u_{t}(x, 0)=0,
$$

and boundary conditions

$$
u(0, t)=0 ; \quad u(\pi, t)=0 ; t \geq 0
$$

applying the double Laplace transform on both sides of (4.13), we have

$$
s^{2} \bar{u}(p, s)-s \bar{u}(p, 0)-\bar{u}_{t}(p, 0)-p^{2} \bar{u}(p, s)+p \bar{u}(0, s)+\bar{u}_{x}(0, s)=0
$$


further application of single Laplace transform to conditions (4.14) and (4.15) gives

$$
\bar{u}(p, 0)=\frac{1}{p^{2}+1} ; \bar{u}_{t}(p, 0)=0 ; \quad \bar{u}(0, s)=0 \text { and } \bar{u}_{x}(0, s)=\frac{s}{s^{2}+1}
$$

Substitute (4.17) in (4.16) and simplify to obtain

$$
\bar{u}(p, s)=\frac{s}{\left(p^{2}+1\right)\left(s^{2}+1\right)}
$$

applying inverse double Laplace transform on (4.18), we obtain

$$
u(x, t)=L_{x}{ }^{-1} L_{t}{ }^{-1}\left\{\frac{s}{\left(p^{2}+1\right)\left(s^{2}+1\right)}\right\}=L_{x}^{-1}\left(\frac{1}{p^{2}+1}\right) L_{t}^{-1}\left(\frac{s}{s^{2}+1}\right)
$$

Thus we obtain the solution of (4.13) as

which is the required solution.

$$
u(x, t)=\sin x \cos t
$$

Example 4.3. Consider the wave equation:

with initial conditions

$$
u_{t t}(x, t)=u_{x x}(x, t)-12 x^{2} ; 0<x<\pi, t>0
$$

and boundary conditions

$$
u(x, 0)=x^{4}+\sin x ; \quad u_{t}(x, 0)=0,
$$

$$
u(0, t)=0 ; \quad u(\pi, t)=\pi^{4}, t \geq 0
$$

applying the double Laplace transform on both sides of (4.21), we have

$$
\begin{gathered}
s^{2} \bar{u}(p, s)-s \bar{u}(p, 0)-\bar{u}_{t}(p, 0)-p^{2} \bar{u}(p, s)+p \bar{u}(0, s)+\bar{u}_{x}(0, s)= \\
-L_{x} L_{t}\left(12 x^{2}\right)
\end{gathered}
$$

further application of single Laplace transform to conditions (4.22) and (4.23) gives

$$
\bar{u}(p, 0)=\frac{4 !}{p^{5}}+\frac{1}{p^{2}+1} ; \quad \bar{u}_{t}(p, 0)=0 ; \quad \bar{u}(0, s)=0 \text { and } \bar{u}_{x}(0, s)=\frac{s}{s^{2}+1}
$$

Substitute (4.25) in (4.24) and simplify to obtain

$$
\bar{u}(p, s)=\frac{s}{\left(p^{2}+1\right)\left(s^{2}+1\right)}+\frac{4 !}{s p^{5}}
$$

applying inverse double Laplace transform on (4.26), we obtain

$$
u(x, t)=L_{x}{ }^{-1} L_{t}^{-1}\left[\frac{s}{\left(p^{2}+1\right)\left(s^{2}+1\right)}\right]+L_{x}^{-1} L_{t}{ }^{-1}\left[\frac{4 !}{s p^{5}}\right]
$$

Thus we obtain the solution of (4.21) as

which is the required solution.

$$
u(x, t)=\sin x \cos t+x^{4}
$$

Example 4.4. Consider the wave equation:

with initial conditions

$$
u_{t t}(x, t)=u_{x x}(x, t)+\sin x ; 0<x<\pi, t>0
$$

$$
u(x, 0)=\sin x ; \quad u_{t}(x, 0)=\sin x,
$$

and boundary conditions

$$
u(0, t)=0 ; \quad u(\pi, t)=0, t \geq 0
$$

applying the double Laplace transform on both sides of (4.29), we have

$$
\begin{gathered}
s^{2} \bar{u}(p, s)-s \bar{u}(p, 0)-\bar{u}_{t}(p, 0)-p^{2} \bar{u}(p, s)+p \bar{u}(0, s)+\bar{u}_{x}(0, s)= \\
L_{x} L_{t}[\sin x]
\end{gathered}
$$


further application of single Laplace transform to conditions (4.30) and (4.31) gives

$$
\bar{u}(p, 0)=\frac{1}{p^{2}+1} ; \quad \bar{u}_{t}(p, 0)=\frac{1}{p^{2}+1} ; \quad \bar{u}(0, s)=0 \text { and } \bar{u}_{x}(0, s)=\frac{1}{s}+\frac{1}{s^{2}+1}
$$

Substitute (4.33) in (4.32) and simplify to obtain

$$
\bar{u}(p, s)=\frac{1}{s\left(p^{2}+1\right)}+\frac{1}{\left(p^{2}+1\right)\left(s^{2}+1\right)}
$$

applying inverse double Laplace transform on (4.34), we obtain

$$
u(x, t)=L_{x}{ }^{-1} L_{t}^{-1} \frac{1}{s\left(p^{2}+1\right)}+L_{x}{ }^{-1} L_{t}{ }^{-1} \frac{1}{\left(p^{2}+1\right)\left(s^{2}+1\right)}
$$

Thus we obtain the solution of (4.29) as

$$
u(x, t)=\sin x+\sin x \sin t
$$

which is the required solution.

Example 4.5. Consider the homogenous wave equation:

with initial conditions

$$
u_{t t}(x, t)=u_{x x}(x, t)-3 u(x, t) ; 0<x<\pi, t>0
$$

and boundary conditions

$$
u(x, 0)=0 ; \quad u_{t}(x, 0)=2 \cos x,
$$

$$
u(0, t)=\sin 2 t ; \quad u(\pi, t)=-\sin 2 t,
$$

applying the double Laplace transform on both sides of (4.37), we have

$$
\begin{gathered}
s^{2} \bar{u}(p, s)-s \bar{u}(p, 0)-\bar{u}_{t}(p, 0)-p^{2} \bar{u}(p, s)+p \bar{u}(0, s)+\bar{u}_{x}(0, s)= \\
-L_{x} L_{t}(3 u)
\end{gathered}
$$

further application of single Laplace transform to conditions (4.38) and (4.39) gives

$$
\bar{u}(p, 0)=0 ; \quad \bar{u}_{t}(p, 0)=\frac{2 p}{p^{2}+1} ; \quad \bar{u}(0, s)=\frac{2}{s^{2}+4} \text { and } \bar{u}_{x}(0, s)=0
$$

Substitute (4.41) in (4.40) and simplify to obtain

$$
\bar{u}(p, s)=\frac{2 p}{\left(p^{2}+1\right)\left(s^{2}+4\right)}+\frac{6 p}{\left(s^{2}-p^{2}\right)\left(p^{2}+1\right)\left(s^{2}+4\right)}-\frac{1}{(s-p)(s+p)} L_{x} L_{t}[3 u]
$$

applying inverse double Laplace transform on (4.42), we obtain

$$
u(x, t)=\cos x \sin 2 t+L_{x}{ }^{-1} L_{t}{ }^{-1}\left\{\frac{1}{(s-p)(s+p)}\left[\frac{6 p}{\left(p^{2}+1\right)\left(s^{2}+4\right)}-L_{x} L_{t}(3 u)\right\}\right.
$$

To apply the Iterative method, we substitute (3.8) in (4.43) and apply (3.12) - (3.14). Thus the components of the solution are:

$$
\begin{gathered}
u_{0}(x, t)=\cos x \sin 2 t \\
u_{1}(x, t)=L_{x}{ }^{-1} L_{t}{ }^{-1}\left\{\frac{1}{s^{2}-p^{2}}\left[\frac{6 p}{\left(p^{2}+1\right)\left(s^{2}+4\right)}-3 L_{x} L_{t}\left(u_{0}(x, t)\right)\right\}=0\right. \\
u_{n+1}(x, t)=L_{x}{ }^{-1} L_{t}{ }^{-1}\left\{\frac{1}{s^{2}-p^{2}}\left[\frac{6 p}{\left(p^{2}+1\right)\left(s^{2}+4\right)}-3 L_{x} L_{t}\left(\sum_{k=0}^{n} u_{k}(x, t)\right)\right\} ; n \geq 1\right. \\
u_{2}(x, t)=L_{x}{ }^{-1} L_{t}{ }^{-1}\left\{\frac{1}{s^{2}-p^{2}}\left[\frac{6 p}{\left(p^{2}+1\right)\left(s^{2}+4\right)}-3 L_{x} L_{t}\left(u_{0}(x, t)\right)\right\}=0\right.
\end{gathered}
$$

Similarly, $u_{3}(x, t)=u_{4}(x, t)=u_{5}(x, t)=0$ and so on.

Thus we obtain the solution of (4.1) as

$$
u(x, t)=\cos x \sin 2 t
$$


which is the required solution.

\section{Discussion of Result}

The aim of the paper is to apply a coupling of double Laplace transform and iterative method to solve linear and non-linear wave equations. This has been demonstrated successfully to get exact solutions to the equations. The iteration process leads to disappearance of noise terms and exact solutions of equations are obtained at first iteration. The simplicity of the method was demonstrated and it doesn't need linearization, perturbation, small parameters etc. for nonlinear operators necessary for some existing techniques.

\section{References}

Al-Jawary, M. A. (2015). Exact solutions to linear and nonlinear wave and diffusion equations. International Journal of Applied Mathematical Research, 4(1), 106-118. https://doi.org/10.14419/ijamr.v4i1.4031

Dhunde, R. R., \& Waghmare, G. L. (2016). Analytical solution of the nonlinear Klein-Gordon Equation using Double Laplace Transform and Iterative method. American Journal of Computational and Applied Mathematics, 6(6), 195-201.

Dhunde, R. R., \& Waghmare, G. L. (2016). Double Laplace Transform combined with iterative method for solving non-linear telegraphic equation. Journal of the Indian Mathematical Society, 83(3-4), 221-230.

Eltayeb, H. (2017). A note on double Laplace decomposition method and nonlinear partial differential equations, New Trends in Mathematical Sciences, NTMCS, 5(4), 156-164. https://doi.org/10.20852/ntmsci.2017.226

Eltayeb, H., \& Kiliciman, A. (2013). A note on double Laplace transform and telegraphic equations. Abstract and Applied Analysis, 2013, 1-6. https://doi.org/10.1155/2013/932578

Sadighi, A., \& Ghanji, D. D. (2007). Exact solutions of nonlinear diffusion equations by variation iteration method. Computer and Mathematics with Applications, 54, 933-939. https://doi.org/10.1016/j.camwa.2006.12.077

Wazwaz, A. M. (2001). Exact solutions to nonlinear diffusion equations by the decomposition method. Applied mathematics \& computation, 123, 109-122. https://doi.org/10.1016/S0096-3003(00)00064-3

Wazwaz, A. M. (2007). The Variational Iteration method: A reliable analytic tool for solving linear and nonlinear wave equations. Computer and Mathematics with Applications, 54, 926-932. https://doi.org/10.1016/j.camwa.2006.12.038

Wazwaz, A. M. (2009). Partial Differential Equations and Solitary Waves Theory ( $1^{\text {st }}$ ed.). New York, NY: Springer. https://doi.org/10.1007/978-3-642-00251-9

\section{Copyrights}

Copyright for this article is retained by the author(s), with first publication rights granted to the journal.

This is an open-access article distributed under the terms and conditions of the Creative Commons Attribution license (http://creativecommons.org/licenses/by/4.0/). 